\title{
Complications of endourological procedures and their treatment
}

\author{
Aldo Franco De Rose ${ }^{1}$, Eugenio Di Grazia ${ }^{2}$, Vincenzo Magnano San Lio $^{3}$, Khaled Refaai ${ }^{4}$, \\ Martina Beverini ${ }^{1}$, Alberto Caviglia ${ }^{1}$, Davide Di Mauro ${ }^{2}$, Giuseppe Giordano ${ }^{3}$, Islam O. Koraiem ${ }^{4}$, \\ Guglielmo Mantica $^{1}$, Diego Meo ${ }^{3}$, Mohamed Ramadan ${ }^{4}$, Mostafa Sakr ${ }^{4}$, Carlo Terrone ${ }^{1}$ \\ ${ }^{1}$ Department of Urology, Policlinico San Martino Hospital, University of Genova, Genova, Italy; \\ ${ }^{2}$ Azienda Policlinico Vittorio Emanuele, Ospedale S. Marco, Catania, Italy; \\ ${ }^{3}$ Unit of Diagnostic and Interventional Radiology ARNAS "Garibaldi-Nesima", Catania, Italy; \\ ${ }^{4}$ Department of Urology, Alexandria University, Egypt.
}

\section{Summary Endourological treatment for urinary stones and other obstructive urinary tract} diseases is minimally invasive but in some cases it involves serious complications. This collection of cases describes some complications of endourological procedures and how they were treated.

Case 1: A case of right ultrasound-guided percutaneous nephrostomy found to be misplaced in the inferior vena cava. The case was safely managed, but it showed that ultrasound guidance alone may be insufficient so it is recommended that percutaneous nephrostomy should be always placed under fluoroscopic control, either alone or in combination with ultrasound guidance.

Case 2: A case of renal subcapsular hematoma occurring on retrograde intrarenal surgery at high perfusion pressure.

The hematoma was drained under combined ultrasonic and radiological guidance. Post treatment recovery was uneventful. Large stone size, severe ipsilateral hydronephrosis, long operation time, higher hydrostatic pressure of the irrigating solution and low ureteral wall compliance are supposed to be risks factors associated with renal subcapsular formation. Management strategy should be tailored to patient's clinical conditions. In hemodynamically stable patients, large hematoma drainage is recommended to prevent further complications and favours early recovery.

Case 3: A case of double J stent fracture discovered one month after the insertion to relieve obstruction from a $1 \mathrm{~cm}$ stone in the right proximal ureter. The distal fragment of the stent was removed by cystoscopy while the proximal fragment was removed by semirigid ureteroscopy in two sessions due to fever and extensive calcification.

Case 4: A mini-invasive technique for transurethral replacement of completely encrusted urinary stents in female patients. This technique allows the interventional radiologist to replace obstructed urinary stents by avoiding more invasive and traumatic urological procedures with sedation.

KEY WORDS: Percutaneous nephrostomy; Inferior vena cava (IVC); Retrograde intrarenal surgery (RIRS); Renal subcapsular hematoma; Ureters; Stents; Ureteral calculi; Fluoroscopy.

Submitted 17 July 2020; Accepted 30 September 2020

\section{Case 1 \\ Misplaced percutaneous nephrostomy tube IN THE INFERIOR VENA CAVA}

Khaled Refaai, Mohamed Ramadan, Islam O. Koraiem, Mostafa Sakr

\section{INTRODUCTION}

Percutaneous nephrostomy (PCN) is a useful tool for temporary drainage of obstructed kidney even with sterile hydronephrosis (1). Depending on the image guidance, PCN can be an effective and safe procedure. However, significant morbidity due to the development of complications can still occur. Potentially serious complications include severe bleeding, septicemia and injury to adjacent organs as bowel perforation, splenic injury, pleural injury or liver injury (2). Major complications rate up to $7 \%$ have been reported. Intravenous misplacement of a urologic catheter is an uncommon complication of percutaneous renal surgery and improper management of it could lead to serious consequences. Different techniques can be used for insertion of PCN. The most commonly used techniques are either Seldinger's technique which include puncture of kidney by a needle then insertion of guide wire and dilatation over this wire to form a wide track enough for passage of nephrostomy catheter or one-stab technique (Bonnano Technique) using a pigtail Teflon catheter mounted on a hollow $18 \mathrm{G}$ needle which has a sharp beveled edge to be directly inserted in the pelvicalyceal system (3). PCN insertion can be either under ultrasound (US) guidance or combined US and fluoroscopy guidance. Less commonly usage of CT can be used especially in abnormal anatomy, ectopic kidney and transplanted kidney (4) Contrast enhanced US can be used in non-dilated kidneys (5). Combined use of ultrasound and fluoroscopy is expected to achieve better results in terms of success rate and decrease in the occurrence of major complications of percutaneous nephrostomy insertion (6). US alone can be used safely in PCN insertion in case of marked dilatation of pelvicalyceal system especially in one stab technique and also can be used in puncture and dilatation in cases of pregnancy or hypersensitivity to contrast material but by well experienced surgeons (3). 


\section{Case report}

A 30-year-old female patient presented to the outpatient clinic with high grade fever and mild right loin pain. Examination revealed mild right loin tenderness. Laboratory blood tests results showed serum creatinine level of $1.5 \mathrm{mg} / \mathrm{dl}$ and white blood cells count of $13.5 \mathrm{x}$ $103 / \mathrm{ml}$ and urine analysis showed pus cells more than 100/HPF. Patient was admitted to hospital and subsequent imaging confirmed the presence of stones in the right kidney with moderate hydronephrosis and thickening of renal pelvis wall reflecting inflammatory stricture. Decision was made to insert a percutaneous nephrostomy (PCN) tube in the right kidney to drain infected hydronephrosis and to ease the later percutaneous nephrolithotomy procedure. Right PCN tube was successfully inserted under ultrasound (US) guidance under cover of broad spectrum antibiotics to be modified after results of urine culture and sensitivity test. Seldinger's technique under US guidance was usually used to insert nephrostomy tube. A 18 gauge $25 \mathrm{~cm}$ long Chiba needle was used to make puncture under US guidance and Teflon dilators starting from 8 to $14 \mathrm{~F}$ over guidewire were used for blind dilatation of the track to insert a $12 \mathrm{~F}$ Amecath nephrostomy tube. Few days later, the PCN tube slipped and decision was made to reinsert the tube. The same urologist reinserted the tube under US guidance following the usual recommended steps. However, after insertion continuous flow of venous blood was unexpectedly noticed. The tube was clamped and fixed and as the patient was vitally stable she was directly transferred to undergo a computed tomography (CT) scan that demonstrated the PCN tube passing within the right renal vein through a segmental renal vein to reach the inferior vena cava (IVC) (Figure 1). The tube was kept clamped and the patient remained vitally stable and decision was made to remove the tube in the operatory room (OR) under vision to be ready for any

\section{Case 1 - Figure 1.}

Computed tomography scan demonstrating nephrostomy tube passing within the right renal vein through a segmental renal vein to reach the inferior vena cava.
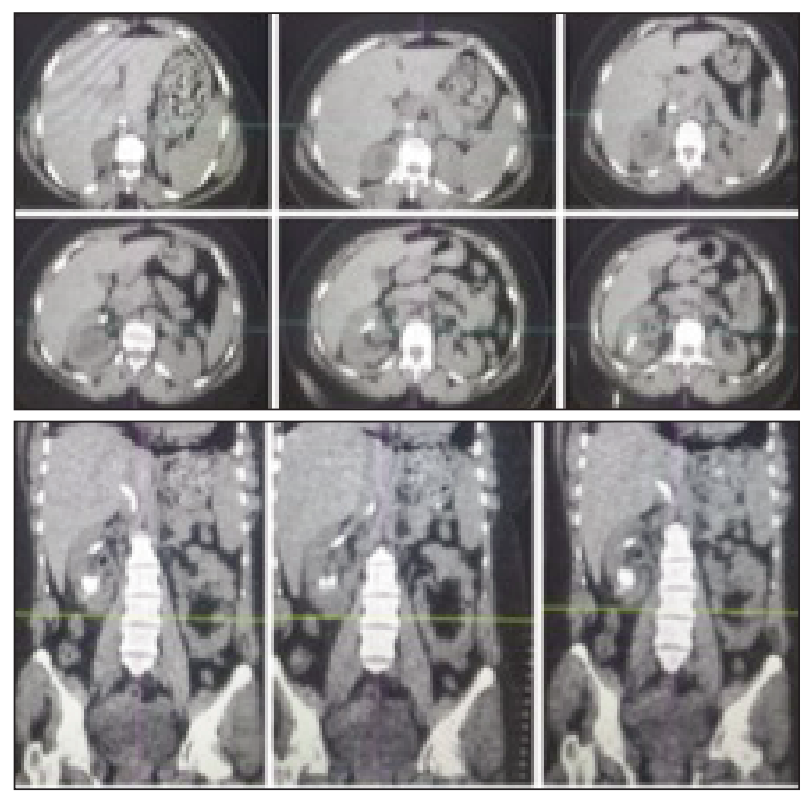

consequences. Exploration was done and PCN was safely removed without any subsequent bleeding, renal stones were extracted through a nephrotomy and double J stent was inserted. On post-operative day 2, patient developed mild respiratory distress. Pulmonary embolism was suspected. However, radiology and laboratory workup was negative. Patient recovered with conservative management and was discharged safely on postoperative day 5 .

\section{Conclusions}

Percutaneous nephrostomy tube should be inserted by welltrained urologists with combined usage of both US and fluoroscopic guidance. In case of intravenous tube misplacement, simple removal is safe and feasible. However, OR team should be ready for any unexpected event.

\section{Case 2 \\ SUBCAPSULAR HEMATOMA AFTER RIRS Eugenio Di Grazia, Davide Di Mauro}

\section{INTRODUCTION}

Retrograde intrarenal surgery (RIRS) is usually safe and effective for managing $<2 \mathrm{~cm}$ kidney stones. Complications rate is generally low and not severe. Major complications occur in fewer than $0.1 \%$ of cases and among these renal subcapsular hematoma (RSH) is exceedingly rare and reported in few papers in literature (7-9). We believe that, although rare, subcapsular hematoma of the kidney deserves attention from endourologists for its severity. We report on a case of renal RSH occurred in a patient after RIRS performed by an experienced endourologist to manage intrarenal kidney stone disease. The presentation, common characteristics, risk factors, management and review of the literature are discussed.

\section{Case report}

A 32-year-old patient had a 3-month long-standing stent deployed at Emergency Unit to relieve left kidney stone obstruction. CT scan before stent placement showed a $1.2 \mathrm{~cm}$ pyelic stone causing hydronephrosis and another $7 \mathrm{~mm}$ stone located at the lower calyx. The patient was scheduled for RIRS. The patient was one pack/day smoker and his body mass index (BMI) was $18.4 \mathrm{Kg} / \mathrm{m}^{2}$.

Preoperative evaluation showed no comorbidities. Urine culture was negative, blood testing showed haemoglobin (Hb) $15.4 \mathrm{~g} / \mathrm{dl}$, white blood count (WBC) 8.520, creatinine $0.78 \mathrm{mg} / \mathrm{dl}$ and normal coagulation tests. After removal of the calcified stent, flexible ureteroscopy (FU) was started with cystoscopic insertion of a hydrophilic safety wire. Then, using a $7 \mathrm{~F}$ semirigid ureteroscope, the ureter was inspected toward the ureteric pelvic junction (UPJ) showing long-standing stent inflammation and no stones along the ureter. We failed to deploy a 10/12 Fr ureteral access sheath (UAS) because of low ureteral wall compliance.

A 7.5 Fr flexible fiberoptic ureteroscope and holmium laser with $200 \mu \mathrm{m}$ fiber were used for lithotripsy. Irrigation was located at $80 \mathrm{~cm}$ from the operating table and manual intermittent forced irrigation with a 20cc syringe was used when necessary to ameliorate visibility. After about 30 minutes operating time the procedure was aborted because of scope friction with the ureter not per- 
mitting to manage the stone properly. A double J stent was replaced and a mini percutaneous nephrolithotomy (miniPCNL) was planned at a later time after appropriate patient counselling. After RIRS, the patient experienced persistent low-grade fever between 37.3 and $38^{\circ} \mathrm{C}$ and a stepwise increase in WBC count in the following days, despite 5 days carbapenems antibiotics full-therapy, and $\mathrm{Hb}$ dropped to $12.5 \mathrm{~g} / \mathrm{dl}$. Other clinical signs such as loin pain or hemodynamic instability were not observed. CT scan showed a $5.6 \times 3.1 \times 11.4 \mathrm{~cm}$ left RSH not supplied by active bleeding (Figure 1). Clinical symptoms and hemodynamic stability induced to manage the complication by placing an $8 \mathrm{~F}$ percutaneous nephrostomy catheter in the urinary system and another drainage in the RSH under ultrasonic and radiological guidance. The previously placed ureteral stent was removed to avoid possible ureteral reflux to the affected kidney during urination.

At first the drainage of the RSH drained about 50cc, then drained about $10 \mathrm{cc}$ per day. Culture test on the aspirate was negative. Drainage ceased to be productive in the fifth day post procedure and it was removed. Post-treatment recovery was uneventful, $\mathrm{Hb}$ stabilized within $10 \mathrm{~g} / \mathrm{dl}$, fever ceased in the second postoperative day and patient was discharged in the fourth postoperative day.

MiniPCNL was performed 2 months later through the same nephrostomy tract when RSH was completely healed. Informed consent was obtained by the patient for the reporting of the case.

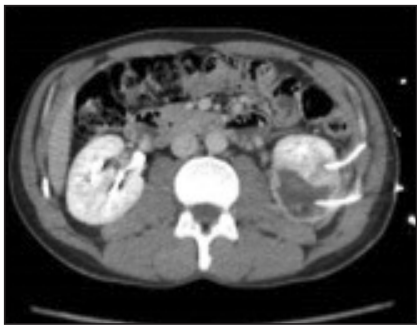

Case 2 - Figure 1.

CT scan showing a $5.6 x$

$3.1 \times 11.4 \mathrm{~cm}$ left renal subcapsular hematoma not supplied by active bleeding.

\section{Conclusions}

After RIRS, RSH is an anecdotal complication caused by fornix rupture and bleeding. Several factors are supposed to favors the risk of renal rupture when high perfusion pressure of irrigation is used. Low pressure irrigation and UAS deployment are recommended to prevent this severe complication. Management strategy is tailored to patient's clinical conditions. In hemodynamically stable patients, large hematoma drainage is recommended to prevent further complications and favors early recovery.

\section{Case 3 \\ RetrieVAl of BROKEn DJ URETERIC STENT \\ A RARE ENDOUROLOGICAL NIGHTMARE \\ Aldo Franco De Rose, Martina Beverini, Alberto Caviglia, Guglielmo Mantica, Carlo Terrone}

\section{INTRODUCTION}

Double-J ureteral stents are commonly used to manage urinary obstructions and their insertion is one of the basic endourological procedure. Even though ureteric stenting is usually a simple and fast maneuver, it is not completely devoid of troubles and complications such as malposition, migration and encrustation. These events require further management that sometimes might be challenging for the endourologist. DJ stent fracture is a possible but rare complication that is reported in literature in very few cases (10-13), mostly related to long time before removal. We present a rare case of DJ stent fracture discovered one month after the insertion.

\section{Case presentation}

In February 2020 a 72 -year-old man presented to another hospital with fever and right renal colic. He performed a CT scan of the abdomen that found a $1 \mathrm{~cm}$ stone in the right proximal ureter, just under the pyelo-ureteral joint. A ureteral double J (DJ) stent was placed. After one month the man was admitted to our first aid with fever and right renal colic. Complete blood count, renal function tests, and serum electrolytes measurement were performed and an increase in white blood cells and creatinine was found (WBC $12.40 \times 10^{\wedge} 9 / \mathrm{L}$, creatinine $2.6 \mathrm{mg} / \mathrm{dL}$, eGFR 24 $\mathrm{ml} / \mathrm{m}^{\prime} / 1.73 \mathrm{mq}$, C-Reactive Protein $41.2 \mathrm{mg} / \mathrm{L}$ ). The urine culture was positive for Staphylococcus Epidermidis and Hominis. He resulted negative at the COVID-19 swab. The patient underwent another abdominal CT scan which showed a right hydronephrosis in addition to the abovementioned stone. Furthermore, the previously positioned DJ stent was broken, with the proximal tip of the stent correctly positioned in the right renal pelvis while the distal half was completely migrated in the bladder (Figure 1A). Despite this, the overall appearance of the right kidney was healthy, with cortical thickness within the limits and presence of moderate perirenal edema. The patient underwent ureterorenoscopy. At the preliminary cystoscopy two fragments of the distal end of the stent were found and were removed with grasping forceps (Figure 1B). After that, the right ureteral meatus was identified and a safety guidewire was positioned. A pyelography with a minimal dose of contrast was done and then a ureteral catheter was advanced along the ureter inducing the push up of the stone. Purulent urine was drained. The semi rigid ureterorenoscope was introduced up to the renal pelvis and the proximal end of the known stent was found there. The stent was taken with a forceps and the removal procedure started, however at the level of the vascular cross the curl of the stent got stuck. Considering the emergency and the septic state of the patient we didn't proceed to laser lithotripsy of the calcified portion of the stent and therefore a mono J urethral stent was placed. After two weeks of

\section{Case 3 - Figure 1.}

A. CT scan showing the broken DJ stent in situ. $B$. DJ stent after the retrieval.

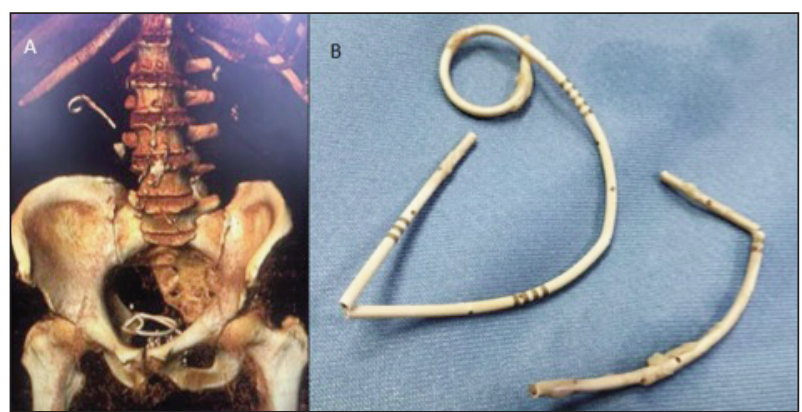


antibiotic therapy, once the phlogistic indexes were normalized, the ureterorenoscopy was repeated and the last portion of the stent and the stone were removed after laser lithotripsy. A double J stent was placed. The patient tolerated the procedure very well and there were no complications. On the following day, the patient was discharged home with the indication to continue the antibiotic therapy and remove the new stent outpatiently a week later.

\section{Conclusions}

Broken DJ ureteric stent is a rare endourological complication. It can be safely removed with minimal morbidity and mortality by an experienced endourologist. To minimize further complications, hospitalization and costs it is preferable, when possible, to perform the removal of the broken DJ stent in one time. It is advisable to perform the surgery in two times in case of complications such as fever or extensive stent calcification.

\section{Case 4}

A MINI-INVASIVE TECHNIQUE FOR TRANSURETHRAL REPLACEMENT OF ENCRUSTED URINARY STENTS IN FEMALE PATIENT. DESCRIPTION OF THE TECHNIQUE AND CLINICAL RESULTS

Vincenzo Magnano San Lio, Diego Meo, Giuseppe Giordano

\section{INTRODUCTION}

Ureteral stent placement is a procedure for maintaining ureteral patency in various benign or malignant conditions (14). It may be a short-term bridge waiting for definitive therapy as in the case of ureteral stones or a long-term stenting for benign or malignant ureteral strictures (15). Plastic stents are the mainstay of long-term treatment. These stents are prone to obstruction and fouling by urinary salt precipitation (15). Traditionally their replacement has been performed cystoscopically, although an alternative is the replacement of urinary stents with a transurethral approach that is performed in the angiography room whose effectiveness is well described in the literature (16). However, complete stent obstruction makes it impossible its replacement on a metal guidewire or an hydrophilic guide wire.

We report the effectiveness of a transurethral recovery technique that allows, while maintaining access to the ureter, to remove the encrusted stent and replace it with an analogue by transurethral technique.

\section{Technique}

The material needed to replace an encrusted ureteral stent is:

- 0,035 inch x 145 Superstiff PTFE coated guidewire (Amplatz, Boston Scientific, Ratingen, Germany)

- 0,035 inch x 180 standard coated hydrophilic guidewire (Radifocus Guidewire M, Terumo, Tokyo, Japan)

- 9 Fr peel away introducer sheath 15,5 cm length or 9 Fr super flex sheath vascular introducer (cutting his terminal valve)

- 3-0 silk thread

- 4 Fr Bern shaped catheter (Bern, Boston Scientific, Ratingen, Germany; Ber, Cordis, Miami, FL, USA)

- Lidocaine Gel 2,5\%
- Klemmer clamp standard or angled

- 8 Fr plastic double J ureteral stent, 20-22-24 cm length (Percuflex, Boston Scientific, Ratingen, Germany)

After accurate disinfection, with a Lidocaine Gel 2,5\% soaked Klemmer clamp the bladder's tip of the ureteral stent is recovered (Figure 1). The proximal portion of the stent (cutting the tip) is externalized and knotted with a silk thread (Figure 2). A metal wire is advanced as far as possible inside the stent until it reaches the obstructed portion and keeping the whole system strained, a coaxial introducer sheath is advanced to reach the ureteral meatus (Figure 3). It is important to do this operation in 2 people, one gently advancing the introducer, the other one holding the catheter under tension through the silk thread. The stent is then removed while maintaining

\section{Case 4 - Figure 1.}

The bladder tip of the ureteral stent was recovered by a jelly soaked Klemmer clamp.
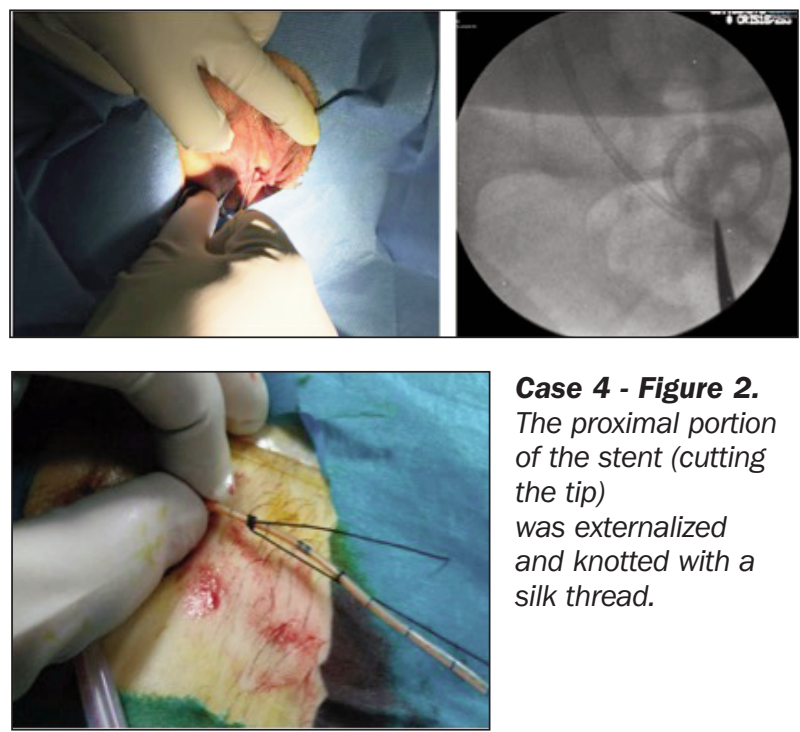

Case 4 - Figure 2.

The proximal portion of the stent (cutting the tip) was externalized and knotted with a silk thread.

Case 4 - Figure 3.

A metal wire was advanced as far as possible inside the stent until it reached the obstructed portion and keeping the whole system strained, a coaxial introducer sheath was advanced to reach the ureteral meatus. The stent was then removed while maintaining access to the ureter.

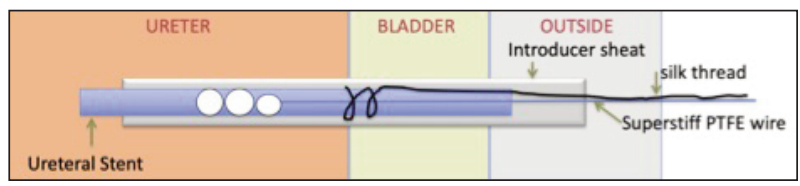

Case 4 - Figure 4.

The renal pelvis was reached with an hydrophilic guide and a 4 Fr Bern catheter. The wire was replaced by a superstiff PTFE coated guidewire. Finally, the new ureteral stent was positioned.

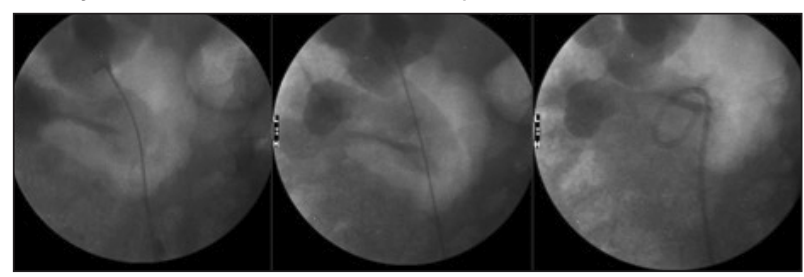


access to the ureter. The renal pelvis is reached, with a hydrophilic guide and a 4 Fr Bern catheter, and the wire is replaced by a superstiff PTFE coated guidewire.

A metal guidewire is advanced up to the renal pelvis inside the introducer sheath and a new $8 \mathrm{Fr}$ stent is positioned (Figure 4). We developed a novel recovery technique with silk thread and use of a 9 Fr vascular introducer sheath which allowed us to remove the obstructed stents while maintaining access to the ureter.

\section{Case series}

From January 2013 to January 2020, 402 urinary stents were replaced with a transurethral approach in 325 women with obstructive urinary disorders (benign and malignant). Out of them 363 were recovered with a standard transurethral approach using a metal guidewire, but 39 stents were obstructed by encrustations making impossible to replace them with a standard technique. In 38 cases it was possible to replace the obstructed stents. The technique failed in one patient because we were unable to advance the access sheath through the ureteral orifice.

Encrusted ureteral stents were replaced in 22 women. The number of procedures performed on each patient, who had an encrusted stent, varied from one to three. Technical success was achieved in $97.4 \%$ procedures. The mean fluoroscopic time was 3 minutes and 25 seconds (range 2 minutes, 31 seconds - 13 minutes, 32 seconds). Twenty patients had urinary infection associated with obstruction due to an occluded stent. There were 6 cases of transient minor hematuria that resolved spontaneously within 1-2 days after the procedure. There were no major complications. Some patients complained of mild pain in the urethra and lower abdomen region when the stent tip was grasped or the stent was removed, but none of them required pain management other than drugs used as part of the pretreatment. In one case a significant stone incrustation in the bladder portion of the stent did not allow for the execution of this technique, therefore we broke the incrustation with the Klemmer clamp once the proximal portion of the stent was externalized and therefore the stent could be replaced.

\section{Conclusions}

In conclusion, fluoroscopically guided transurethral replacement of encrusted ureteral stents in women is a feasible and safe alternative to cystoscopy. Performing this exchange procedure reduces costs compared to cystoscopic exchange of the ureteral stent in the operating room. This technique allows to replace obstructed urinary stents avoiding more invasive and traumatic urological procedures with sedation.

\section{References}

1. Mittal V, Biswas M, Lal S. Percutaneous nephrostomy or double $J$ stenting, which is better modality for obstructive uropathy-a descriptive study. Int J Res Med Sci. 2017; 4:3486-91.

2. Dyer RB, Assimos DG, Regan JD. Update on interventional uroradiology. Urol Clin North Am. 1997; 24:623-52.

\footnotetext{
Note:

Supplementary Discussion and References

are published online as Supplementary Materials at

https://www.pagepressjournals.org/index.php/aiua/index
}

3. Venyo A, Fatola C, Adegbite D, Khan A. Nephrostomy in pregnancy a district general hospital experience over five years. Journal of Biomedical Graphics and Computin. 2014; 5:10.5430/jbgc.v5n1p1.

4. Ghani KR, Patel U, Anson K. Computed tomography for percutaneous renal access. J Endourol. 2009; 23:1633-9.

5. Liu BX, Huang GL, Xie XH, et al. Radiology. 2017; 285:293-301.

6. Bhatt S, Verma P, Grover Ret al. Success, effectiveness and safety of combined sonographic and fluoroscopic guided percutaneous nephrostomy in malignant ureteral obstruction. Int J Radiol Radiat Ther. 2017; 3:165-70.

7. Yahsi S, Tonyali S, Ceylan C, et al. Intraparenchymal hematoma as a late complication of retrograde intrarenal surgery. International Braz J Urol. 2017; 43:367-370.

8. Kozminski MA, Kozminski DJ, Roberts WW, et al. Symptomatic subcapsular and perinephric hematoma following ureteroscopic lithotripsy for renal calculi. Journal of Endourology. 2015; 29:277-282.

9. Bai J, Li C, Wang S, et al. Subcapsular renal haematoma after holmium:yttrium-aluminum-garnet laser ureterolithotripsy. BJU International. 2012; 109:1230-1234.

10. Chua ME, Morales ML Jr. Spontaneous fracture of indwelling polyurethane ureteral stents: A case series and review of literature. Can Urol Assoc J. 2012; 6:386-392.

11. Ray RP, Mahapatra RS, Mondal PP, Pal DK. Long-term complications of JJ stent and its management: A 5 years review. Urol Ann. 2015; 7:41-45.

12. El-Faqih SR, Shamsuddin AB, Chakrabarti A, et al. (1991) Polyurethane internal ureteral stents in treatment of stone patients: morbidity related to indwelling times. J Urol. 146:1487-1491.

13. Zisman A, Siegel YI, Siegmann A, Lindner A. Spontaneous ureteral stent fragmentation. J Urol. 1995; 153 (3 Pt 1):718-721.

14. Park SW, Cha IH, Hong SJ, et al. Fluoroscopy-guided transurethral removal and exchange of ureteral stents in female patients: technical notes. J Vasc Interv Radiol. 2007; 18:251-6.

15. McCarthy E, Kavanagh J, McKernan S, et al. Fluoroscopically guided transurethral removal and/or replacement of ureteric stents in women. Acta Radiol. 2015; 56:635-40.

16. Carrafiello G, Coppola A, De Marchi G, et al. Trans-Urethral Ureteral Stent Replacement Technique (TRUST): 10-Year Experience in 1168 Patients. Cardiovasc Intervent Radiol. 2018; 41:610-617.

\section{Correspondence}

Aldo Franco De Rose, MD - aldofrancoderose@libero.it

Martina Beverini, MD (Corresponding Author) - martina.beverini@live.it

Alberto Caviglia, MD - caviglialberto@gmail.com

Guglielmo Mantica, MD - guglielmo.mantica@gmail.com

Carlo Terrone, MD - carlo.terrone@med.uniupo.it

Department of Urology, Policlinico San Martino Hospital, University of Genova

Largo Rosanna Benzi 10, 16132 Genova

Davide Di Mauro, MD (Corresponding Author) - davidedimauro84@virgilio.it

Eugenio Di Grazia, MD - eugeniodigrazia@hotmail.com

Azienda Policlinico Vittorio Emanuele, Ospedale S. Marco, Catania (Italy)

Magnano San Lio Vincenzo, MD

Meo Diego, MD (Corresponding Author) - diegomeo@hotmail.it

Giordano Giuseppe, MD

Unit of Diagnostic and Interventional Radiology ARNAS "Garibaldi-Nesima"

Via Palermo 636, 95122 Catania (Italy)

Khaled Refaai, MD - javacup16@gmail.com

Mohamed Ramadan, MD

Islam O. Koraiem, MD (Corresponding Author) - drislamosama@live.com Mostafa Sakr, MD

Uro-oncology Unit, Department of Genito-Urinary Surgery, Faculty of Medicine, Alexandria University 74 Albert st, Smouha, 5359472, Alexandria, Egypt 